\title{
Éditorial
}

\section{Propulser la promotion de la santé et la prévention des maladies chroniques hors des marges des plans d'action en santé publique globale - nouvelle tentative}

\author{
Robert Geneau, Ph. D., rédacteur scientifique en chef, Revue PSPMC
}

Un an après le début de la pandémie de coronavirus, et avec la campagne de vaccination maintenant en cours, l'espoir d'un retour progressif à une vie " normale » grandit de jour en jour. Toutefois, le véritable portrait de cette normalité est incertain, comme en témoigne le flot constant de publications dans lesquelles on s'interroge sur ce que seront nos vies après la COVID-19, qu'il s'agisse de l'avenir du travail de bureau ou des changements dans la façon dont nous aménagerons et construirons nos villes, offrirons des services de santé et ferons face aux changements climatiques et aux inégalités économiques. « Rebâtir en mieux » est devenu une expression couramment utilisée par les gouvernements et les organismes internationaux ${ }^{1,2}$.

On espère que ce mantra sera appliqué aux systèmes de santé publique, en partie grâce à une diplomatie scientifique plus vigoureuse à l'échelle mondiale et, souhaitons-le, à de nouveaux investissements systématiques dans la prévention des maladies après la COVID-19. On appelle au respect des principes de "santé publique globale $»^{3}$, on propose d'appliquer et de mettre en œuvre une approche " une seule santé $»^{4}$ et de favoriser davantage la pensée intégrative à propos de la manière dont la pandémie actuelle est liée à d'autres problèmes de santé et aux principaux déterminants de la santé ${ }^{5}$.

Dans ce contexte, certains proposent que la COVID-19 soit considérée comme un phénomène syndémique* plutôt que pandémique, affirmant que "les affections se regroupent au sein de groupes sociaux en calquant les inégalités profondément enracinées dans nos sociétés » et que dans le cas de la COVID-19, " s'attaquer [aux maladies chroniques] sera une condition préalable à la réussite d'un confinement » [traduction] $]^{7, p .874}$. Dans son plus récent rapport, l'administratrice en chef de la santé publique du Canada a souligné la nécessité d'adopter une approche plus ferme en matière d'équité dans le domaine de la santé pour lutter contre la COVID-19 et elle a fait remarquer que « la pandémie de la COVID-19 a ébranlé notre conscience collective et nous a amenés à reconnaître que l'équité est essentielle pour assurer la sécurité sanitaire $»^{8, \mathrm{p} .2}$.

Mais ceci a comme un air de déjà-vu. Depuis la première réunion de haut niveau des Nations unies sur les maladies non transmissibles (MNT) en 2011, les appels visant à renouveler notre approche à l'égard des MNT et à mettre en œuvre un programme de santé globale plus intégré se sont intensifiés. Les progrès demeurent lents. En 2018, dans son ouvrage intitulé Il est temps d'agir : Rapport de la Commission indépendante de haut niveau sur les maladies non transmissibles, l'Organisation mondiale de la santé a conclu qu'à l'échelle mondiale, nous ne sommes pas en voie de réduire d'un tiers la mortalité prématurée due aux MNT d'ici 2030 (objectif de développement durable 3 , cible 3.4$)^{9}$. Les commissaires ont souligné qu'" un sentiment de normalité persiste, au lieu de l'urgence qui devrait s'imposer »", p.4 et que, de ce fait, les investissements nationaux et internationaux ont été insuffisants.
Si la COVID-19 doit apporter une nouvelle perspective ou un nouveau sentiment d'urgence pour faire face aux maladies chroniques et aux principaux déterminants de la santé, quels principes ou stratégies pourraient guider nos prochains pas? Voici ce qui pourrait constituer un point de départ.

- Nous devons reformuler les discours sur les maladies chroniques et leurs causes sous-jacentes et éliminer la division artificielle entre maladies chroniques et maladies infectieuses. Il est clair que le chemin va être long. C'est un fait bien connu, du moins chez les scientifiques, que les objectifs de développement durable des Nations unies sont profondément interreliés. Des investissements accrus dans la prévention et le contrôle des maladies infectieuses - investissements qui sont nécessaires - ne devraient pas entraîner une diminution du financement visant d'autres affections ou visant les principaux déterminants de la santé. Adopter une approche à somme nulle constituerait un manque de vision. Promouvoir et utiliser le terme "syndémique » pour décrire la COVID-19 pourrait nous faire avancer un peu plus.

- Nous avons besoin de meilleures données. Cette affirmation doit être précisée. Même si nous disposons de données qui montrent que les maladies chroniques sont l'un des facteurs critiques des décès dus à la COVID-19 et qu'elles illustrent la gravité de la

* Le modèle syndémique de la santé met l'accent sur l'ensemble biologique et social, qui regroupe les maladies interactives, coexistantes et séquentielles et les facteurs sociaux et environnementaux qui favorisent et amplifient les effets négatifs de l'interaction entre les maladies ${ }^{6}$.

Rattachement de l'auteur :

Agence de la santé publique du Canada, Ottawa (Ontario), Canada

Correspondance : Robert Geneau, Agence de la santé publique du Canada, 785, rue Carling, Ottawa (Ontario) K1A 0K9; courriel : PHAC.HPCDP.Journal-Revue.PSPMC.ASPC@canada.ca 
maladie, il n'y a pas assez de données désagrégées de qualité sur les populations vulnérables (p. ex. absence de données fondées sur l'origine ethnique) pour éclairer les décisions. L'étude de l'incidence plus générale de la COVID-19, qui inclut les conséquences (santé mentale, consommation problématique de substances, etc.) des mesures prises en santé publique, a pleinement montré l'importance des études longitudinales pour répondre aux questions de recherche complexes. Une sensibilisation accrue aux lacunes actuelles pourrait donner l'impulsion nécessaire pour s'y attaquer de front.

- $\quad$ Nous ne pouvons permettre à la quête d'innovation de nous distraire des démarches éprouvées en matière de santé publique. Bien que nous ayons manifestement besoin de vaccins pour nous aider à sortir de la crise actuelle de la COVID-19 - et nous devrions célébrer l'innovation dans le domaine des vaccins à l'ère de la COVID-19 - il importe de rappeler que les vaccins sont un outil essentiel mais ne constituent pas une solution miracle ${ }^{10}$. Les mêmes conseils sont valables dans le domaine de la promotion de la santé et de la prévention des maladies chroniques. Une foule de stratégies fondées sur la technologie mobile - sous l'égide de l'« innovation » - ont été utilisées pour aider les gens à améliorer leurs comportements en matière de santé. Ces stratégies relèvent d'une trousse d'outils mais ne doivent pas se substituer à l'adoption de politiques fondées sur des données probantes pour prévenir les maladies chroniques. Il arrive parfois qu'on mette l'accent sur les comportements et la responsabilité des individus pour détourner l'attention et éviter de s'occuper des déterminants de la santé structurels ou plus en amont.

- Nous devons nous orienter vers les solutions. La crise de la COVID-19 se prête à des études expérimentales naturelles, par exemple sur les effets des interventions non pharmaceutiques sur l'incidence de la COVID-19, les effets à grande échelle des mesures de santé publique ou encore les effets des interventions visant à atténuer les conséquences négatives de ces mesures. Les expériences naturelles sont encore un outil sous-utilisé pour améliorer les données probantes en santé publique.
La grande majorité des manuscrits soumis à la revue PSPMC visent à décrire le «problème » - les mesures du fardeau et du coût économique de la maladie, etc. - et cela revêt évidemment une importance cruciale. Ce qui manque encore, ce sont des études plus rigoureuses sur l'impact des expériences naturelles et des interventions à l'échelle de la population. Les efforts de recherche en santé publique à l'ère de la COVID-19 pourraient contribuer à solidifier le domaine de la recherche interventionnelle en santé des populations.

Il ne s'agit ici aucunement d'une évaluation exhaustive de la manière de s'assurer que le domaine de la promotion de la santé et de la prévention des maladies chroniques évolue au sein d'un mouvement plus vaste visant à renforcer les systèmes de santé publique. La série d'articles de la revue PSPMC sur la COVID-19 (avec un modèle de publication en ligne d'abord) demeure ouverte. Nous invitons de nouveau nos collègues du Canada à nous envoyer des manuscrits sur la COVID-19, qu'il s'agisse d'éditoriaux ou de commentaires prospectifs ou encore d'article de recherche originaux sur les conséquences à grande échelle des mesures de santé publique.

\section{Références}

1. Bakker P, Elkinton J. To build back better, we must reinvent capitalism. Here's how [Internet]. Genève, Suisse : Forum économique mondial, 2020 [consulté le 25 janvier 2021]. En ligne à : https://www.weforum.org/agenda /2020/07/to-build-back-better-we -must-reinvent-capitalism-heres-how/

2. Ministère des Finances Canada. Rebâtir en mieux : Un plan pour lutter contre la récession causée par la COVID-19 [Internet]. Ottawa (Ontario) : Gouvernement du Canada; 2020 [consulté le 25 janvier 2021]. En ligne à : https:// budget.gc.ca/fes-eea/2020/themes /building-back-better-rebatir-mieux-fr .html

3. Loewenson R, Accoe K, Bajpai N, et al. Reclaiming comprehensive public health. BMJ Glob Health. 2020;5(9): e003886. https://doi.org/10.1136/bmjgh $-2020-003886$
4. Ruckert A, Zinszer K, Zarowsky C, Labonté R, Carabin $\mathrm{H}$. What role for One Health in the COVID-19 pandemic? Can J Public Health. 2020;111(5): 641-644. https://doi.org/10.17269 /s41997-020-00409-z

5. The Lancet. COVID-19: a new lens for non-communicable diseases. Lancet. 2020;396(10252):649. https://doi.org /10.1016/S0140-6736(20)31856-0. Erratum dans : Lancet. 2020;396(10254): 818. https://doi.org/10.1016/S0140-6736 (20)31902-4

6. Singer $M$, Bulled $N$, Ostrach $B$, Mendenhall E. Syndemics and the biosocial conception of health. Lancet. 2017;389(10072):941-950. https://doi .org/10.1016/S0140-6736(17)30003-X

7. Horton R. Offline: COVID-19 is not a pandemic. Lancet. 2020;396(10255): 874. https://doi.org/10.1016/S0140 $-6736(20) 32000-6$

8. Agence de la santé publique du Canada. Du risque à la résilience : Une approche axée sur l'équité concernant la COVID-19. Ottawa (Ont.) : Gouvernement du Canada; 2020. 88 p. [ $\mathrm{n}^{\circ}$ au catalogue : HP2-10E-PDF]

9. Organisation mondiale de la santé (OMS). Il est temps d'agir : Rapport de la Commission indépendante de haut niveau sur les maladies non transmissibles. Genève (Suisse) : OMS; 2018. En ligne à : https://apps.who .int/iris/handle/10665/274339

10. BBC World News Coronavirus : WHO warns of 'no silver bullet' amid vaccine search [Internet]. Londres : BBC; 2020. En ligne à : https://www.bbc .com/news/world-53643455 